\title{
TRASTORNOS POR SUSTANCIAS PSICOTRÓPICAS Y NULIDAD MATRIMONIAL*
}

\author{
MENTAL DISORDERS CAUSED BY PSYCHOTROPIC SUBSTANCES \\ AND MARRIAGE ANNULMENT
}

\section{BLANCA LIRA CORREA**}

RESUMEN: El objetivo de la ponencia fue: 1. Examinar la aceptación y el traspaso a categorías jurídicas de los estudios realizados por la psicología y psiquiatría, en el ámbito del derecho canónico. 2. Evaluar la incidencia de los trastornos por sustancias psicotrópicas en la capacidad de los contrayentes para el consentimiento matrimonial. 3. Dar a conocer nuevas conductas de la mujer en relación con las sustancias psicotrópicas, y áreas que deben ser evaluadas y estudiadas, al momento de analizar la capacidad para discernir el matrimonio, o asumir/cumplir los derechos y obligaciones que este implica.

Palabras clave: Matrimonio, sustancias psicotrópicas, nulidad matrimonial, derechos/ obligaciones matrimoniales, discreción de juicio.

ABSTRACT: the object to this presentation is to 1. Examine the acceptance of psicologic studies into the canonic law. 2. to evaluate the importance of quimical substances into the capacity to a person who is signing a marriage contract. 3. expose the new conducts of women related to quimical substances in areas that should be evaluated and studied when we analyze the capacity to understand the rights and obligations inside the marriage contract.

Key words: Matrimony, Quimical substances, marriage annulment, rights and obligations derivate from a marriage contract.

El Papa Pío XII en la inauguración del año judicial de 1941 (3 de octubre), aplaude una sentencia de la Rota Romana, coram Wynen, "por servirse de los progresos de las ciencias psicológicas y psiquiátricas" $\mathrm{y}$ anima a los jueces a asimilar estas nuevas corrientes, siempre que se tratase de verdadera ciencia y no de vagas teorías ${ }^{2}$. Con este mensaje se inició una de las aventuras intelectuales más apasionantes del siglo XX, cual fue traspasar a categorías jurídicas los avances de la psiquiatría y la psicología.

Al mismo tiempo se comenzó a evolucionar en el derecho canónico -base para las actuales causales de nulidad matrimonial civil- hacia un carácter personalista del matrimonio, tendencia que conlleva una visión integral del ser humano, tanto en su aspecto

* Ponencia efectuada con motivo de la Inauguración del año judicial del Tribunal Eclesiástico, año 2010.

** Abogado y Magíster en Ciencia Jurídica de la Pontificia Universidad Católica de Chile, Jueza del Tribunal Eclesiástico de Santiago. Dirección postal: Camino Otoñal 1655, Las Condes, Chile. Teléfonos 2245404/09-2333400. Correo electrónico: blanca_lira_c@hotmail.com

${ }^{1}$ BACCIOLI (2003) pp. 153-154.

2 PÍO XII (1941) en: Enchiridion Familiae, p. 1026. 
físico, intelectual, espiritual, psicológico, etc. Por esto en materia de nulidad matrimonial, se han desarrollado una serie de áreas en las que resulta necesario profundizar en búsqueda de la verdad.

Este artículo tiene por objeto aproximarse a un problema de gran relevancia en la sociedad actual, y por consiguiente en nuestros tribunales. Se trata de los trastornos por sustancias psicotrópicas, y los efectos que estos producen en la personalidad de quienes consumen dichas sustancias, en orden a su capacidad parta consentir en matrimonio.

Antes de entrar de lleno en el tema, resulta relevante destacar, que el mismo título constituye una novedad, por cuanto ya no nos referimos a alcoholismo y drogadicción, o drogodependencia o toxicomanías, como se ha hecho tradicionalmente, sino a "trastornos por sustancias psicotrópicas". Esto es así, puesto que es la forma como se las describe actualmente en los manuales de psiquiatría, y dice relación con la ingestión de drogas de abuso, tales como la marihuana y cocaína, y se incluyen también en esta terminología el alcohol, medicamentos y la exposición a tóxicos ${ }^{3}$. Además, esta es la terminología que se utiliza en las últimas sentencias rotales, siguiendo el lineamiento del Manual de Psiquiatría DSM-IV.

Es a mi juicio necesaria la adopción de esta nueva terminología, puesto que para que se produzca consenso entre juristas y psicólogos, se deben homogeneizar los términos utilizados paralelamente en el derecho y otras ciencias, porque opiniones y terminologías diferentes causan confusión y desorientan.

Por otra parte más de alguna sentencia rotal ha criticado la clásica distinción doctrinaria y jurisprudencial canónica, relativa al alcoholismo y drogadicción. Actualmente no se usa en psiquiatría clasificación alguna de las drogas, sino que se agrupan en bloques de acuerdo con los trastornos mentales que ellas producen ${ }^{4}$.

Finalmente, cabe tener presente que aun cuando en el ámbito del derecho canónico se trate habitualmente en forma independiente al alcoholismo de la drogadicción o toxicomanías, "los principios generales son comunes" 5 en ambas.

\section{EVIDENTE INTERRELACIÓN ENTRE LOS JUECES CANÓNICOS Y LAS CIENCIAS HUMANAS}

Puesto que algunas de las causales de nulidad tienen su fundamento en una incapacidad de naturaleza psíquica, y en virtud de que los trastornos por sustancias psicotrópicas, constan de diversas etapas cuya gravedad y antigüedad resultan muy difíciles de determinar para el juez, este deberá recurrir a los informes psicológicos y psiquiátricos, realizados en conformidad a los principios inspiradores del derecho civil o canónico, según cuál sea la disciplina que aborde el problema.

El juez necesita del perito para que le facilite los elementos concernientes a su específica competencia, "es decir, la naturaleza y el grado de las realidades psíquicas o

\footnotetext{
3 DSM-IV-TR (2002) p. 217.

4 SENdín BlázQueZ, Juan Agustín (2004) pp. 248-249.

${ }^{5}$ MARTÍNEZ (2000) p. 584.
} 
psiquiátricas" ", pero no trasvasando simplemente conceptos médicos en la jurisprudencia sino que debe hacer una adaptación de estos, a los principios que rigen el derecho matrimonial. Una vez que el perito otorga al juez el diagnóstico propio de su ciencia, es el juez quien debe detectar las formas más graves de anomalías que incapaciten a una persona para contraer matrimonio, y le impidan otorgar un válido consentimiento matrimonial. Para poder realizar esta operación, el juez debe conocer los presupuestos científicos y técnicos del trastorno del que se trate.

Para este efecto, quienes se desempeñan en los Tribunales Eclesiásticos o de Familia, deberían utilizar como base para el análisis de esta problemática, el Manual de Psiquiatría DSM-IV ${ }^{7}$, por cuanto contiene una mejor sistematización de los trastornos relacionados con sustancias, y es de más fácil comprensión para el lector. También resulta de gran ayuda el CIE- $10^{8}$, manual que contiene la clasificación internacional de las enfermedades psiquiátricas, elaborado por la Organización Mundial de la Salud?

El DSM-IV, a nuestro juicio, es un manual más preciso y útil para quienes no tienen dominio de las ciencias psicológicas y psiquiátricas, debido a que hace una descripción de cada una de las sustancias, y las consecuencias en la personalidad del sujeto según el grado de adicción o consumo. Puesto que cada sustancia psicotrópica tiene efectos peculiares en la personalidad de quien la consume, "a la Jurisprudencia le interesa conocer los efectos de la droga en general y los específicos de cada una de ellas en orden a determinar la capacidad del sujeto para contraer"lo.

La razón por la que sugerimos el uso de estos manuales es porque, tal como señala el juez rotal, Juan Agustín Sendín ${ }^{11}$, en la Jurisprudencia canónica, fuente fundamental para nuestro estudio, "existe una aceptación sin reticencia de los estudios de la OMS" y "en relación con el Manual diagnóstico y estadistico de los trastornos mentales DSM-IV, hemos observado una progresiva referencia primero, hasta llegar a una aceptación total en las últimas sentencias".

\section{TRASTORNOS POR SUSTANCIAS COMO BASE PARA LAS CAUSALES DE NULIDAD MATRIMONIAL}

Debido a que en el matrimonio - más que en cualquier otro negocio jurídico- las personas se comprometen en toda su integralidad, y los trastornos por sustancias psicotrópicas producen un detrimento de la salud física y mental de quienes los padecen, estos trastornos pueden constituirse en base para el análisis de las causas de nulidad matrimonial.

\footnotetext{
${ }^{6}$ BACCIOLI, Carlos (2004) vol. XI, p. 208.

7 DSM-IV-tr (2005) Pp. 217-332 (Manual de Diagnóstico y estadístico de los Trastornos MENTALES).

${ }^{8}$ CIE-10 (1992) p. 424.

${ }^{9}$ CIE-10 (1992) pp. 97 y ss.

${ }^{10}$ Martínez (2000) p. 589.

${ }^{11}$ SENDÍn (2004) pp. 281-282.
} 
En el Derecho Canónico, tanto la doctrina como la Jurisprudencia han abordado directamente los efectos que dichas patologías producen, y su influencia perturbadora en la capacidad de consentir, lo que ha permitido a su vez consolidar el recurso a las ciencias que estudian al ser humano, desde el derecho.

Por ello, no obstante el progreso de las ciencias en esta materia, no podemos ignorar los aportes de la jurisprudencia canónica. En efecto, fue en una coram Sabattani de 24 de febrero de $1961^{12}$, donde por primera vez se trató específicamente el tema del alcoholismo en materia de nulidad matrimonial, la que arrojó como aporte, la distinción entre alcoholismo agudo y crónico, y una escala de criterios para determinar la gravedad del alcoholismo. Posteriormente sentencias como la coram Bruno de 18 de diciembre de $1986^{13}$ o la coram Sendín Blázquez de 23 de julio de $2001^{14}$, por nombrar algunas, han sido de gran ayuda en el establecimiento de criterios útiles en la traducción de la información psicológica y psiquiátrica a categorías jurídicas, en esta área ${ }^{15}$.

La descripción de los efectos producidos por sustancias en quienes las consumen, contenidas en estos estudios, son de gran ayuda para que los jueces puedan obtener la certeza moral requerida para dictar sentencia, al momento de contrastar todas las pruebas obtenidas en el proceso. Es el juez quien "debe valorar desde la perspectiva jurídica las enseñanzas psiquiátricas y psicológicas y en su virtud subsumir el caso concreto" 16 en alguno de los supuestos legales mencionados.

\section{ETAPAS Y GRAVEDAD DE LOS TRASTORNOS POR SUSTANCIAS:}

La doctrina canónica sugiere que se comprueben dos aspectos básicos para determinar si los trastornos por sustancias pueden ser causa de incapacidad. Por una parte, la antigüedad y etapa del proceso tóxico y por otra, la gravedad de la perturbación ${ }^{17}$, de tal modo que estos trastornos tengan, a la fecha de la celebración del matrimonio, una entidad suficiente para impedir al sujeto establecer la comunidad de vida y amor que requiere el matrimonio. Si el trastorno por sustancias aparece después de la celebración del matrimonio, no podemos afirmar que existe nulidad matrimonial.

El perito debe demostrar que el sujeto sufría de una intoxicación aguda, o una intoxicación crónica, al momento de contraer matrimonio, que dicha adicción era capaz de producir un trastorno de personalidad en el contrayente, y que este trastorno se encontraba presente, al menos en forma latente, al momento de consentir. Pero será el juez quien con los demás antecedentes del proceso deberá determinar si dicho trastorno incapacitó al sujeto para hacerse cargo como dueño y titular responsable de los deberes y obligaciones del matrimonio.

\footnotetext{
12 Coram Sabattani, 24 de febrero de 1961, p. 123.

13 Coram Bruno de 18 de diciembre de 1986, p. 759.

14 Coram Sendín Blázquez de 23 de julio del 2001 en: REDC, p. 879.

15 Mendonça (2000), vol. CXXV, II, p. 366.

16 Martínez (2000) p. 586.

17 Coram Stankiewicz, en Ius Canonicum, p. 287.
} 
En cuanto a la etapa del proceso se debe distinguir entre el consumo, la intoxicación y la dependencia de sustancias psicotrópicas, puesto que las dinámicas y consecuencias que estas producen, van a variar según la etapa en que el sujeto se encuentre.

La dependencia o intoxicación crónica como se le ha llamado en el ámbito del derecho canónico, indica que el sujeto continúa consumiendo la sustancia a pesar de la aparición de problemas significativos relacionados con ella, ya sea en el área cognoscitiva, de comportamiento o fisiológica. Una de las características de esta etapa es que los intereses del adicto comienzan a quedar influidos de manera más o menos importante, por el vínculo del sujeto con la sustancia, la que pasa a ocupar un sitial privilegiado en el psiquismo, y la vida del sujeto comienza a girar en torno a la obtención de la sustancia ${ }^{18}$.

En estos casos los miembros del tribunal deberán tener en consideración, la gravedad de la perturbación, y si esta tuvo la entidad suficiente, según la sustancia psicotrópica de que se trate, como para impedir al contrayente que sufre el trastorno, asumir y cumplir las obligaciones matrimoniales o autodeterminarse en torno a la decisión matrimonial.

Si se está frente a una intoxicación aguda, esta corresponde a una condición mental transitoria causada por una ingestión reciente de sustancias psicotrópicas. Aquí el juez deberá tener en consideración el tipo de sustancia consumida, la cantidad, las características especiales del contrayente consumidor, el grado de alteración psíquica que esa intoxicación produjo en el contrayente al momento de la celebración del matrimonio, para determinar si esta sustancia le impidió entender o captar algo ajeno a la droga, o por el contrario, le permitió deliberar y hacer un acto libre y elegir algo que no sea su droga, y comprometerse en una entrega generosa al otro cónyuge.

El gran aporte de la Jurisprudencia canónica, dada su observación en casos concretos, son los criterios desarrollados para determinar el consumo por sustancias, que produzcan en el contrayente tal dependencia, que ya no tenga dominio sobre sí mismo, coartando su libertad, o su capacidad de asumir los derechos-obligaciones matrimoniales. En este sentido recomiendan a todos quienes trabajamos en los Tribunales Eclesiásticos lo siguiente ${ }^{19}$ :

- Utilización de los criterios creados por Sabattani.

- Valoración del comportamiento de quien sufre el trastorno por sustancias desde el principio, teniendo en cuenta las condiciones psíquicas personales del contrayente

- Afirmación de que la verdadera prueba ha de deducirse del conjunto de los hechos pre y postnupciales

- Valoración de la antigüedad del proceso tóxico

- Podemos agregar un quinto criterio, cual es el análisis de la sustancia consumida y sus efectos en el caso concreto

${ }^{18}$ LÓPeZ, Cristián (2006) p. 67.

${ }^{19}$ Coram Sendín Blázquez de 23 de julio de 2001, p. 880. 


\section{TRASTORNOS POR SUSTANCIAS PSICOTRÓPICAS EN CHILE}

Los trastornos por sustancias, se han convertido en problemas de gran relevancia en nuestra sociedad, por su incidencia en el matrimonio y la vida familiar y social.

$\mathrm{Al}$ estudiar los trastornos por sustancias psicotrópicas, pretendemos contribuir a aportar a quienes trabajan en el Tribunal Eclesiástico e incluso los Tribunales de Familia, elementos que sirven de base para el desarrollo de capítulos de nulidad matrimonial, y subsumir estos trastornos en las normas legales que contienen las incapacidades para otorgar el consentimiento matrimonial, contempladas en el canon 1095 del Código de Derecho Canónico, y en el artículo $5^{\circ}$ de la Nueva Ley de Matrimonio Civil ${ }^{20}$, relativo a la nulidad, que tomó como modelo inspirador para su formulación, al Derecho Canónico.

En efecto, el consumo, abuso y dependencia de sustancias psicotrópicas, tales como marihuana, alcohol y cocaína entre la juventud en Chile, ha crecido sostenidamente desde el año 2004 y no así en la población adulta, en donde la tendencia es a disminuir ${ }^{21}$. En el último estudio del CONACE se señala por ejemplo, que el consumo de marihuana entre los jóvenes alcanza un 25\% y que uno de cada cuatro de estos jóvenes muestran síntomas de dependencia. Lo anterior implica problemas en el área familiar, laboral, la integridad física, e incluso problemas con la ley.

Dado el significativo número de adolescentes -futuros contrayentes del vínculo matrimonial- involucrados en el consumo de sustancias psicotrópicas, y los efectos que estas producen en ellos a nivel neuropsicológico ${ }^{22}$, especialmente en la región prefrontal del cerebro, que tiene "repercusiones en funciones cognitivas relevantes como la planificación, toma de decisiones, control comportamental, memoria de trabajo o procesos de atención, entre otros" 23 , surge la necesidad de analizar la capacidad para consentir en matrimonio de jóvenes cuya ingesta se inició a temprana edad.

\section{NUEVOS ASPECTOS A CONSIDERAR DADA LA EXPERIENCIA EN LAS CAUSAS DE NULIDAD}

\section{Alcoholismo femenino:}

El consumo de sustancias psicotrópicas en mujeres ha cambiado considerablemente. Hasta hace pocos años, el consumo femenino se realizaba en la clandestinidad, quienes consumían se avergonzaban, y existía un rechazo social al consumo femenino.

\footnotetext{
${ }^{20}$ Ley 19.947, Nueva Ley de Matrimonio Civil. Diario Oficial de 17 de mayo de 2004.

21 www.bibliodrogas.cl/biblioteca/digital/octavo_estudio_nacional_2008_final.pdf, p. 16

22 Concluyen los autores, que el alcohol puede alterar tanto la estructura como la función del sistema nervioso central, de manera significativa. Por su parte el consumo crónico de alcohol puede ocasionar deterioro neurológico y cognitivo, siendo una de las áreas afectadas, la que dice relación con las funciones ejecutivas. Las funciones ejecutivas son aquellas "que dicen relación con los procesos mentales que ponemos en marcha para resolver problemas generados interna y externamente”. GARCÍA MORENO, Luis (2007) en: www.adicciones.es/revista-new.php?register_vais_[nume]=73, p. 272.

23 CADAVEIRA (2009) p. 11.
} 
Actualmente las mujeres han incrementado el consumo de sustancias psicotrópicas, especialmente las jóvenes, lo hacen públicamente, y su consumo se ha equiparado al de los hombres ${ }^{24}$. Atendido lo anterior, estimo relevante hacer hincapié en la necesidad de que quienes trabajamos en el tribunal eclesiástico, evaluemos los efectos que este consumo produce en orden a la capacidad para asumir/cumplir las obligaciones matrimoniales.

Con el objeto de graficar la urgencia del tema, cabe señalar por ejemplo, que diversos estudios realizados en madres alcohólicas concluyen que sus hijos poseen ciertas características comunes, tales como: "alteraciones cráneo-faciales, retraso en el crecimiento, hiperactividad, retraso mental y alteraciones psicomotoras entre otras" 25 , afectaciones que serán proporcionales al grado de alcoholismo de la madre. Estos niños tienen altas probabilidades de desarrollar SAF (Síndrome alcohólico fetal) que implica para el niño consecuencias a nivel intelectual, neurológico y de comportamiento.

Incluso "la ingesta de pequeñas cantidades de alcohol durante la gestación puede conducir a alteraciones en la formación del sistema nervioso fetal que se manifiestan con déficit cognitivo"26. Lo mismo sucede con otras sustancias psicotrópicas y las consecuencias van a variar, dependiendo del tipo de sustancia, la cantidad consumida, y el tiempo de gestación. Así, algunas sustancias producen efectos en las habilidades perceptivas del niño, o en el control de sus estados conductuales, o en el desarrollo social y emocional y en las funciones motoras, entre otras ${ }^{27}$.

Estos aspectos son relevantes en orden al matrimonio, debido a que una vez que se ha establecido el abuso, por ejemplo del alcohol, la dependencia alcohólica en las mujeres, progresa con mayor rapidez que en los hombres, dadas sus condiciones físicas ${ }^{28}$. Esta realidad deberá tenerse en cuenta en orden a considerar la dependencia por sustancias ya sea en acto próximo, o presente al momento del consentimiento, y de qué modo estos trastornos por sustancias afectan la capacidad de la mujer-madre para disponerse al bien de los cónyuges o al bien de la prole, hacia los que se ordena el matrimonio. En caso que el juez concluya que la mujer con un trastorno de personalidad es incapaz de asumir estos bienes, esa incapacidad deberá reconducirse al canon 1095, 2 ó 3 del Código de Derecho Canónico, o a las causales de nulidad establecidas en el artículo 5 de la Ley 19.947, Nueva Ley de Matrimonio Civil

\section{AfEctación del núcleo familiar de UnA PERSONA QUE SUfre TRASTORNOS POR SUSTANCIAS PSICOTRÓPICAS:}

Porque es toda la familia nuclear y extendida, de quienes sufren un trastorno por sustancias psicotrópicas, quienes se ven directamente afectados por este problema debido a que son causantes de mayor incidencia de muertes prematuras, episodios de violencia, crisis familiares y violaciones. Este tema es de gran relevancia, y alguna doctrina ha

\footnotetext{
${ }^{24}$ www.bibliodrogas.cl/biblioteca/digital/octavo_estudio_nacional_2008_final.pdf, p. 122.

25 GUERri, C. (2000) pp. 147-154.

26 GUERri, C. op. cit., p. 155.

27 Calvo Botella, Helena (2003) en: www.adicciones.es/files/Calvo.pdf

28 Coram Sendín Blázquez de 23 de julio del 2001, p. 879.
} 
destacado que la Jurisprudencia canónica no le ha dado mayor énfasis a la inhabilidad de los padres que sufren un trastorno por sustancias, en orden a asumir las obligaciones esenciales del matrimonio ${ }^{29}$.

Cualidades como el altruismo, el sacrificio personal, autocontrol, responsabilidad y estabilidad necesarios para sostener una comunidad de vida familiar no son compatibles con un estilo de vida con episodios de abuso o dependencia de sustancias psicotrópicas. Los cónyuges-padres afectados por un trastorno por sustancias son generalmente agresivos, inmaduros, irresponsables, egocéntricos, tienen un sentido moral debilitado, son impulsivos y celosos, elementos que, según nuestra vasta experiencia en los tribunales, dejan huellas profundas en los hijos y el otro cónyuge, que repercute en sus propias decisiones y relaciones matrimoniales adultas. Son familias que generalmente crecen con sensación de vergüenza, culpa, soledad y perfeccionismo, y los hijos habitualmente tienen riesgo de tener alteraciones conductuales, psicológicas y cognitivas, tales como la adopción de roles inflexibles como una forma de sobrevivencia emocional ${ }^{30}$.

Por tanto los jueces debemos considerar que no solo quienes tienen trastornos por sustancias pueden ser incapaces para consentir en matrimonio, sino también la prole puede verse afectada por una futura incapacidad, puesto que no pudieron obtener en su hogar de origen la seguridad, orden, afectos, soporte y guía, requeridas para un desarrollo armónico de la personalidad.

\section{CONCLUSIÓN}

- El aumento del consumo de sustancias psicotrópicas es una realidad que no podemos ignorar, básicamente porque sus consecuencias afectan directamente a la familia, base fundamental de nuestra sociedad.

- Los trastornos de personalidad producidos por el abuso o dependencia de sustancias psicotrópicas, pueden constituirse en base para declarar la nulidad de un matrimonio determinado, si es que estas tuvieron una entidad tal que incapacitaron al contrayente para realizar un juicio crítico valorativo, o le impidieron su capacidad de autodeterminarse, o de asumir/cumplir las obligaciones matrimoniales.

- Es preciso destacar la importancia que tiene la formación complementaria de los jueces de los Tribunales Eclesiásticos y de Familia en otros ámbitos, que no es el

\footnotetext{
${ }^{29}$ MORRIS, Patrick (1999) p. 228 "Jurisprudence has not placed significant emphasis on the alcoholic or the non-alcoholic individual's inability to parent a a ground for incapacity to assume essential abligations... a jugje could combine evidence of irresponsable behavior, on the part of one who abuses alcohol, prior to consent, with evidence of parental neglect of children subsequent to consent and arrive at moral certitude regardin the inhability to assume this essential obligation".

${ }^{30}$ MORRIS, Patrick (1999) pp. 227-230 "The modeling of the alcoholic parent is obviusly inadequate and the process of identification is, therefore, impaired. The alcohol abuse, behavioural problems, and accademic difficulties of children and adolescents from alcoholic homes all may give witness to de disorganization and neglect characteristic of families centred around alcohol. In addition, children of alcoholic adopt inflexible roles in the service of emotional survival and are, therefore, often ill equipped to meet the demand of later adult adjustment". "The altruism, self-sacrifice, control, responsibility, continuity and stability necessary to sustain a commitment are not compatible with the alcohol abuser's lifestyle".
} 
exclusivamente jurídico, puesto que sin un conocimiento científico y técnico mínimo, difícilmente podrán llegar a una conclusión razonada, y hacer una valoración libre, pero crítica de las pruebas y pericias presentadas, en un proceso de nulidad matrimonial.

- Por otra parte el conocimiento, unido a la experiencia en el análisis de las conductas humanas y matrimoniales, solo contribuyen al bienestar de la sociedad en la medida que se dan a conocer, por lo que su desarrollo constituye para quienes trabajamos en los tribunales, una misión pedagógica y de aporte intelectual, que no podemos ignorar.

\section{BIBLIOGRAFÍA}

BACCIOLI, Carlos (2004): "Puntos fundamentales de la pericia psicológico-psiquiátrica en el proceso de nulidad matrimonial" en: Anuario Argentino de Derecho Canónico" (Buenos Aires, Pontificia Universidad Católica Argentina) vol. XI año 2004, pp. $185-210$.

(2003): "Los aportes de la Medicina, la Psiquiatría y la Psicología al Derecho Canónico" en: Anuario Argentino de Derecho Canónico (Buenos Aires, Anuario Argentino de Derecho Canónico) vol. X año 2003, pp. 139-160.

Cie-10 (1992): Trastornos mentales y del comportamiento. Descripciones clinicas y pautas para el diagnóstico (Editor español J.J. López-Ibor, Madrid, Meditor) p. 424.

GUERRI, C. (2000): "Malformaciones en el desarrollo inducidas por el consumo materno de alcohol durante la gestación” en: Nuevas interpretaciones y nuevos abordajes del alcoholismo" (Bilbao, Instituto de Deusto) pp. 147-154.

DSM-IV-TR (2005): Manual diagnóstico y estadístico de los trastornos mentales (Director de la versión española: Juan J. López-Ibor, Barcelona, Masson) p. 1049.

LÓPEZ, Cristián (2006): "La adicción a sustancias químicas ¿puede ser efectivo un abordaje psicoanalítico?” en: Psyke (Santiago, Lom Ediciones) vol. $15 \mathrm{~N}^{\circ}$ 1, mayo 2006, p. 67

MARTínez Blanco, Antonio (2000): "Reflexiones sobre la incidencia de la drogadicción en el consentimiento matrimonial canónico según la jurisprudencia de la Rota Romana (1986-1994)" en: Hominum causa omne ius constitutum est. Escritos sobre el matrimonio en homenaje al Prof. Dr. José María Díaz Moreno, S.J. (Castán Vázquez, J.M. et al., Coordinadores, Madrid, Universidad Pontificia de Comillas) pp. 582-615.

MEndonçA, Augustine (2000): “The effects of alcohol-related disorders on matrimonial consent” en: Monitor Ecclesiasticus (Roma, Fondazione Monitor Eccelsiasticus) vol. CXXV, III, pp. 550-600.

MORRIS, Patrick (1999): "Alcoholism and marital consent: an investigation of the alcoholic capacity/incapacity to Exchange valid consent" (London, The Catholic Marriage Tribunal).

PíO XII (1941) en: Enchiridion Familiae, textos sobre el Magisterio Pontificio y Conciliar sobre el matrimonio y la familia (siglos I al XX) (editado por Augusto Sarmiento, Universidad de Navarra, Pamplona), vol. II. 
SENDín BLÁZqueZ, Juan Agustín (2004): “Intoxicación y dependencia de sustancias -alcoholismo y drogadicción- como causas de falta de suficiente uso de razón, grave defecto de discreción de juicio e incapacidad de asumir y cumplir las obligaciones esenciales del matrimonio en la jurisprudencia de la Rota Romana” en: Curso de derecho matrimonial y procesal canónico para profesionales del foro, vol. XVI (Coordinado por Myriam Cortés, Salamanca, Publicaciones Universidad Pontificia) pp. 239-365.

\section{SENTENCIAS CITADAS}

(18 de Diciembre de 1986) Coram Bruno (grave falta de discreción de juicio por falta de libertad) en: ARRT vol. 78, 1991, $\mathrm{N}^{\circ} 7$.

(24 de febrero de 1961) Coram Sabattani (grave defecto de discreción de juicio) en: Sacrae Romanae Rotae Decisiones seu Sententiae.

(22 febrero 1996) Coram Stankiewicz: Tribunal de la Rota Romana (Nulidad de matrimonio, exclusión e incapacidad para asumir las obligaciones matrimoniales) en: Ius Canonicum (Pamplona, Universidad de Navarra. Instituto Martín de Azpilcueta) vol. XXXIX No 77 año 1999, pp. 259-303.

(23 de julio 2001) Coram Sendín Blázquez: Tribunal de la Diócesis de Plasencia (Nulidad de Matrimonio, Defecto de Discreción de juicio e incapacidad para asumir las obligaciones) en: Revista Española de Derecho Canónico (Salamanca, Pontificia Universidad de Salamanca) vol. $61 \mathrm{~N}^{\circ} 157$ pp. 837-951.

\section{RECURSOS ELECTRÓNICOS}

Calvo Botella, Helena (2003) "Maternidad, infancia y drogas: implicaciones clínicas" [Fecha de Consulta: 27 de abril de 2010] Disponible en: www.adicciones.es/files/ Calvo.pdf

www.bibliodrogas.cl/biblioteca/digital/octavo_estudio_nacional_2008_final.pdf, p. 122 [Fecha de Consulta: 21 de abril de 2010.

García Moreno, Luis (2007) "Actividad prefrontal y alcoholismo de fin de semana en los jóvenes" p. 272 en: www.adicciones.es/revista-new.php?register_vais_[nume]=73 